\title{
Technical note: Use of an automated grooming brush by heifers and potential for radiofrequency identification-based measurements of this behavior
}

\author{
Rachel L. Toaff-Rosenstein, ${ }^{*}$ Martin Velez, $\dagger$ and Cassandra B. Tucker ${ }^{1 *}$ \\ ${ }^{*}$ Center for Animal Welfare, Department of Animal Science, and \\ †Department of Computer Science, University of California, Davis 95616
}

\begin{abstract}
Healthy cattle readily use grooming brushes but this behavior subsides when animals become ill. Tracking use of a brush may provide an opportunity for health monitoring, especially if the process could be automated. We assessed how healthy heifers groom themselves on a brush and hypothesized that radiofrequency identification (RFID) could be used to accurately and automatically record this behavior. Angus and Hereford heifers $(\mathrm{n}=16)$ were fitted with 2 ultra-high-frequency RFID ear tags and monitored in groups of 8 while housed in a pen with an electronic brush, video cameras, and 4 RFID antennas. Each heifer was observed for a 6 -h period using continuous video recordings, and brush contact was characterized in terms of anatomic region involved (head/neck, trunk, or posterior) and when not touching the brush but within 1 body length of it. The RFID data were collected for the same period and then processed such that intervals of up to $16 \mathrm{~s}$ with no detections but contained between 2 recordings were also considered positive (animal in brush proximity). Brush proximity (RFID) was regressed against brush contact duration (video) and the sensitivity and specificity for each individual heifer calculated. Across heifers, the majority of brush use involved the head/ neck, although a few heifers demonstrated relatively large amounts of posterior contact, which contributed to false-negative readings when antennas failed to read the ear tags. Furthermore, for the majority of time that animals were near the brush, they were not in contact with it but rather standing or lying nearby, resulting in false-positive readings. It follows that the ability of the RFID system to accurately detect brush contact varied widely across individual heifers (sensitivity 0.54-1.0; specificity 0.59-0.98), with RFID generally overestimating the duration of brush proximity relative to actual time spent in brush contact. The implication
\end{abstract}

Received April 4, 2017.

Accepted June 19, 2017.

${ }^{1}$ Corresponding author: cbtucker@ucdavis.edu is that RFID-based ear tag recording of brush proximity relative to continuous video observations of contact does not yield accurate results in certain heifers and therefore, as currently configured, is not a reliable representation of this type of grooming behavior.

Key words: automation, grooming, health monitoring, radiofrequency identification (RFID)

\section{Technical Note}

Grooming brushes enable self-care behaviors (e.g., hair-coat maintenance) when cattle are kept in relatively barren environments (as reviewed by Wilson et al., 2002; DeVries et al., 2007; Mandel et al., 2016). Monitoring grooming may facilitate health assessment (Weary et al., 2009), whereby sick animals, including cattle, groom less than healthy counterparts as part of a strategy in which energetic resources are redirected away from behaviors that are not essential in the short term and instead toward enhanced immune function (Hart, 1988; Borderas et al., 2008; Toaff-Rosenstein et al., 2016). Stress, including high ambient temperatures, manipulations such as AI, and calf removal in dairy cows, may also induce changes in brush use (Mandel et al., 2013; Newby et al., 2013), though whether this behavior increases or decreases appears to be situationdependent.

Manual recording of animal behavior is laborious and generally impractical for commercial use. In contrast, automatic behavior recording may enable more timely and accurate large-scale sickness detection and welfare assessment (e.g., as reviewed in swine; Matthews et al., 2016). Specific examples include halters (Büchel and Sundrum, 2014) to monitor feeding, collars to track rumination (Ambriz-Vilchis et al., 2015), and computerized video analysis of activity levels (Ott et al., 2014). Radiofrequency identification (RFID) is another technology that can be used to monitor behavior automatically. In a common RFID setup, powered antennas (or readers) continuously transmit wireless interrogator radio waves. This signal powers nearby tags, which decode the signal and transmit back their unique serial number. The antennas then pass this information to a computer 
for data storage and processing. High-frequency RFID systems enable reading of multiple tags simultaneously, which is beneficial when tracking numerous animals. Radiofrequency identification has shown promise when used with electronic (gated) feed and water stations (Chapinal et al., 2007) to monitor dairy cow eating and drinking behavior. It has also successfully recorded the presence of beef cattle at an ungated feed bunk, a proxy for feeding (Mendes et al., 2011). However, RFID was less successful in recording pig feeding (Maselyne et al., 2014) and mice exploratory behaviors (Catarinucci et al., 2014).

Our objectives were to (1) describe how healthy heifers use a brush in terms of body region groomed, on a proportional basis, and (2) evaluate whether RFIDbased determination of brush proximity is an accurate substitute for video observations of contact, indicating a role for this technology in automatically recording this behavior. We hypothesized that RFID-determined brush proximity could serve as a suitable substitute for video-determined brush contact and therefore enable accurate, automatic recordings of this type of grooming behavior.

The University of California, Davis Animal Care and Use Committee reviewed and approved all procedures used (Protocol 16947). Data collection was completed between May 30 and June 2, 2014, at the University of California, Davis beef research facility (Davis, CA). Heifers ( 8 Angus and 8 Hereford) between the ages of 7 and 9 mo with an initial average BW of $269 \mathrm{~kg}$ (range 198 to $364 \mathrm{~kg}$ ), originating from the university cow-calf herd and subjected to clinical examination by a veterinarian to verify health status, were enrolled. Heifers were randomly assigned to 1 of 2 groups, balanced for breed and BW, and managed per standard herd health protocol, including treatment with a topical ectoparasiticide and use of fly-repellant ear tags. Their diet consisted of $47.5 \%$ flaked corn, $17.2 \%$ dried distillers grains, $13.7 \%$ alfalfa hay, $11.7 \%$ oat hay, $7.1 \%$ molasses, and $1.3 \%$ fat and containing $87.5 \%$ DM, $13.3 \%$ $\mathrm{CP}, 27.7 \% \mathrm{NDF}$, and $75.8 \% \mathrm{TDN}$, and providing 1.8 Mcal of $\mathrm{NE}_{\mathrm{M}} / \mathrm{kg}$ and 1.2 Mcal of $\mathrm{NE}_{\mathrm{G}} / \mathrm{kg}$. Water was provided ad libitum from a self-filling trough.

Ten days before commencement of data collection, heifers were individually restrained in a chute for approximately $30 \mathrm{~min}$ and each marked with unique numerical identification (left and right withers and flank) using hair dye (Clairol Nice 'N Easy Borne Blonde; Procter and Gamble, Cincinnati, OH). Additionally, the distal portion of the tail switch was trimmed, leaving approximately $5 \mathrm{~cm}$, to avoid entrapment of the tail in the mechanical brush (described below), per the manufacturer's recommendation.
Heifers were transported $3 \mathrm{~km}$ to the trial site and housed by group ( $\mathrm{n}=8 /$ group), with brush exposure beginning $3 \mathrm{~d}$ before start of data collection. Data collection occurred in a covered, concrete-floored, Lshaped pen, which was enclosed but allowed for dim natural lighting and ventilation. The dimensions of the area containing the brush and feed bunks were 9.1 $\times 7.3 \mathrm{~m}$. An automated grooming brush (model no. 91526202 Swinging Cow Brush, DeLaval, Kansas City, $\mathrm{MO}$ ) was installed on the wall, $0.81 \mathrm{~m}$ above the floor measured from the bottom of the brush, according to manufacturer instructions (Figure 1). The brush $(90 \times$ $90 \times 82 \mathrm{~cm}$ ) hung vertically from a pivoting arm from which it could move in many directions, including over the animal's back, when manipulated. When an animal pushed the brush off center to a $\geq 30^{\circ}$ angle, it began rotating at a speed of 26 rotations/min. Rotation continued until $10 \mathrm{~s}$ after returning to a vertical position, after the heifer was no longer pushing the brush. The lying area measured $9.1 \times 4.9 \mathrm{~m}$, including a $4.9-\times$ 4.3-m bedded area with a $\sim 25$-cm-deep layer of straw.

Black and white CCTV video cameras (model no. WV-BP334, Panasonic Corporation of North America, Secaucus, NJ) and lenses (model no. 13VG2812ASII, Tamron, Commack, NY) were connected to a digital video recorder with digital surveillance software (GeoVision Surveillance System V8.5 Inc., Taipei, Taiwan). To enable video recording during low-light conditions, red holiday lights suspended from pen rafters operated on a timer between 1600 and $0800 \mathrm{~h}$. Eight cameras focused on the brush (suspended 2.0 to $2.2 \mathrm{~m}$ above). Each camera was set to continuously record at medium quality and 30 frames/s.

An RFID reader (ALR-9900+, Alien Technology, Morgan Hill, CA) was attached to the wall of the pen above the brush. Four 25- $\times 25$-cm panel antennas (model no. S9028PCL96RTN, Laird USA, Earth City, $\mathrm{MO}$ ) were mounted $2 \mathrm{~m}$ above the ground from wood beams attached to the rafters, in each quadrant around the brush, at a distance ranging from 0.6 to $1.3 \mathrm{~m}$ from its center (Figure 1, marked with asterisks), and set to an attenuation level of 25 to confine the reading range to a relatively small radius around the brush. Ultra-high-frequency RFID chips (ALN-9629, Alien Technology, Morgan Hill, CA) were attached to each existing left and right plastic identification ear tag using a single layer of duct tape.

Data were collected from the 2 groups sequentially for a total of $6 \mathrm{~h} /$ group, between 0600 and $1200 \mathrm{~h}$. All $6 \mathrm{~h}$ of data were collected on a single day from the first group. Following this, the second group was moved into the vacated pen and the $6 \mathrm{~h}$ of data collected over the course of $2 \mathrm{~d}$. 
Continuous video observations of brush-related behaviors (Table 1) were recorded to the nearest second of when they began and ended. Specifically, all physical contact with the brush was considered grooming and characterized in terms of anatomic region involved. Then, the proportion of total contact involving the head/neck, trunk, and posterior was calculated. A semi-rigid wire, shaped into a circle and attached to the camera housing, was used to approximate an animal body length, so that video observers could determine whether the animal was near the brush, even when not making physical contact (Figure 2). Specifically, standing near the brush while not in brush contact was differentiated from lying. The proportion of time in which the heifer was in brush contact versus standing or lying nearby was calculated.
The computer software, a Java 7 program written using the Alien Software Development Kit (v2.3.1; Alien Technology), communicated with the RFID reader via the network. Every second, the RFID reader sent data to the software about which tags were sensed by the antennas. The software then parsed and stored the data on local and remote PostgreSQL 9.3 databases. A web application, built using Ruby-on-Rails 4, provided real-time views of the data and implemented functions to assist in processing and analyzing the data. Data were post-processed such that intervals (up to a maximum of $16 \mathrm{~s}$ ) with no RFID detections but contained between 2 readings were considered positive for the entire duration (animal in brush proximity). This decision was made because it was found to yield the highest sensitivity and specificity relative to video-

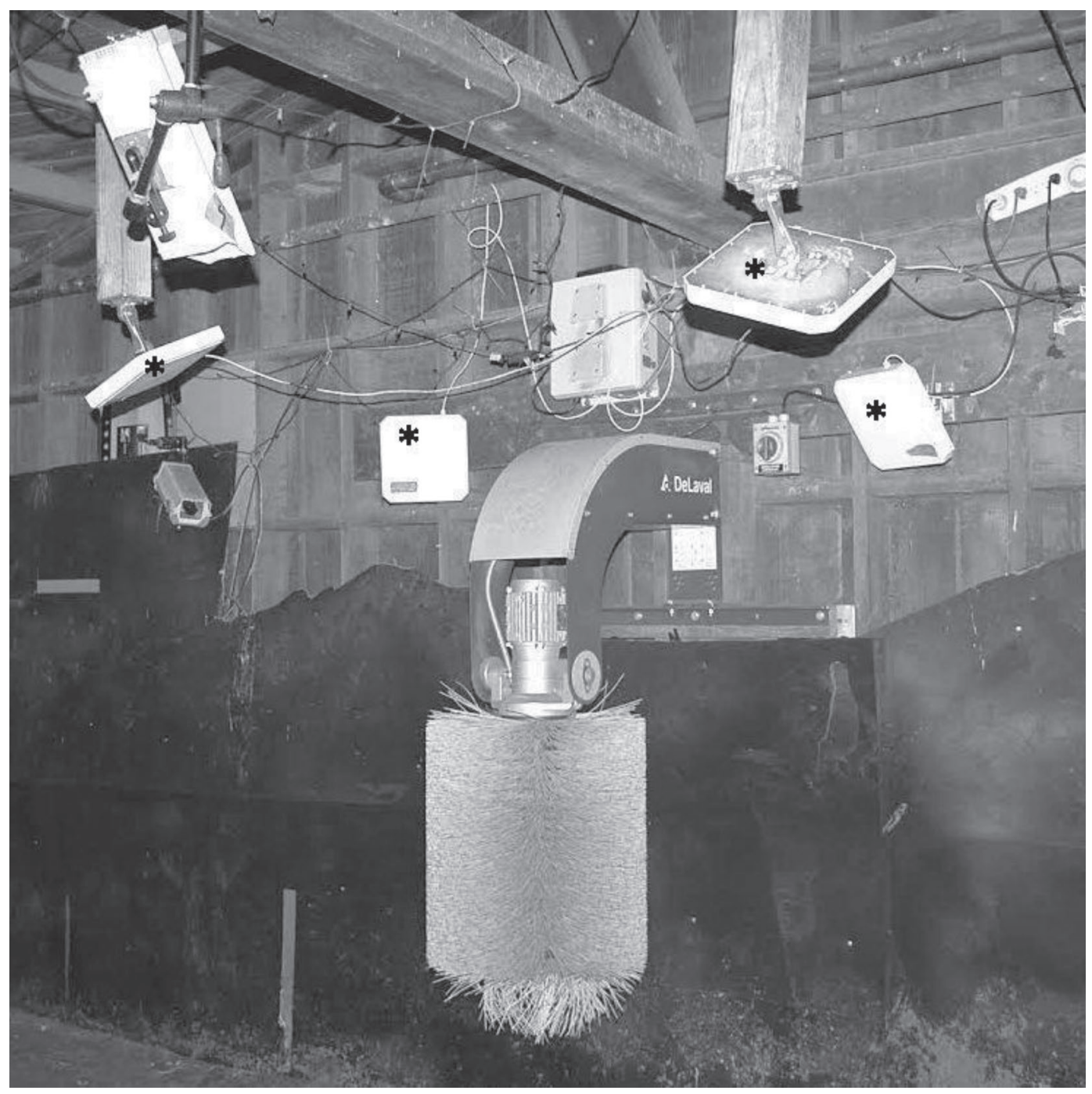

Figure 1. The installation of the automated grooming brush, radiofrequency identification (RFID) reader (on wall above brush), and 4 antennas, each marked with an asterisk $(*)$. Several of the video cameras installed around the brush are also visible. 
Table 1. Ethogram used during video observations of cattle use of a brush; each behavior is mutually exclusive

\begin{tabular}{ll}
\hline Behavior relative to a brush & Description \\
\hline Head/neck contact ${ }^{1,2}$ & Brush bristles are primarily touching between the muzzle and start of the shoulders \\
Trunk contact & Brush bristles are primarily touching between and including the shoulders/forelimbs and up to but \\
excluding the narrowest point in front of the hips & Brush bristles are primarily touching the hind limbs, rump or tail, beginning at the narrowest point in \\
Posterior contact ${ }^{1,2}$ & front of the hips \\
& Heifer has no physical contact with the brush bristles but at least part of animal is within a body length \\
Standing nearby & from them \\
& Heifer, whose weight is supported by the trunk of the body, has no contact with brush bristles but at \\
Lying nearby & least part of the animal is within a body length from them
\end{tabular}

${ }^{1}$ Weight is supported by 2 or more limbs; typically, the animal is standing (weight supported by 4 limbs) but may also be in process of getting up or lying down.

${ }^{2}$ Times are within $\pm 1.0 \mathrm{~s}$ of when the behavior begins and ends.

${ }^{3}$ The partial circle (indicated with the black arrow in Figure 2) approximates an animal body length. Two separate overhead cameras with an attached wire were used to make this determination. If an animal appeared within the partial circle according to either or both cameras, it was considered in brush vicinity.

recorded brush contact after analyzing data using various post-processing schemes.

Inter- and intra-observer reliability for the 5 observers was calculated using PROC REG (SAS software version 9.4, 2014; SAS Institute Inc., Cary, NC). Interobserver reliability had an $\mathrm{R}^{2} \geq 0.91$, an intercept of $0(P \geq 0.50)$, and slope of $1(P \geq 0.32)$; intra-observer reliability had an $\mathrm{R}^{2} \geq 0.88$, intercept of $0(P \geq 0.15)$, and slope of $1(P \geq 0.20)$.

The ability of RFID to measure grooming behavior was evaluated by examining the relationship between duration of brush contact according to video compared

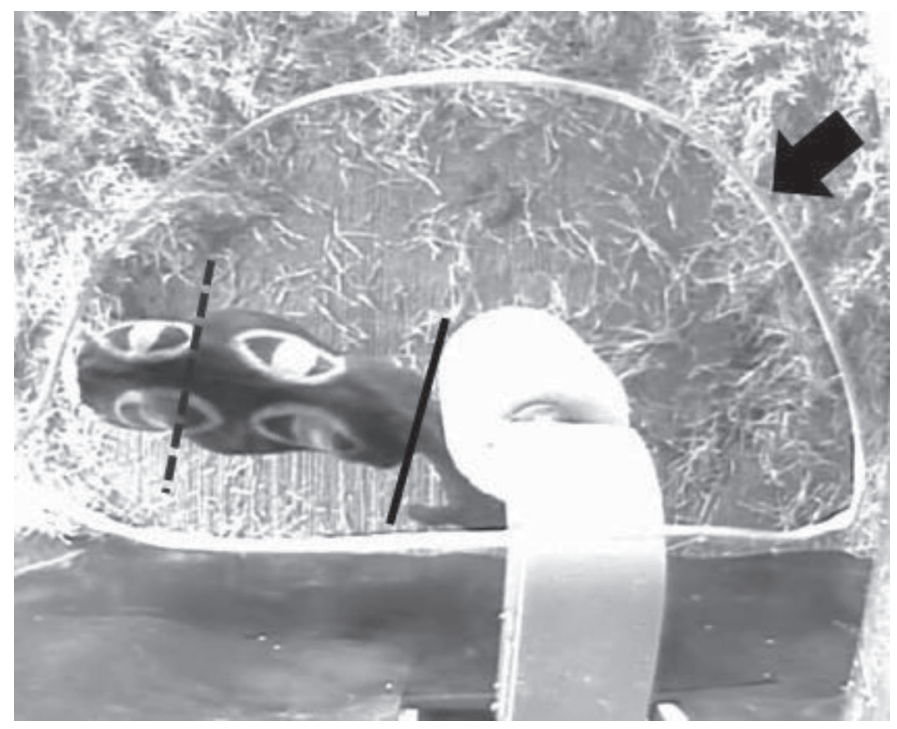

Figure 2. Animal performing head/neck contact with a brush. The solid black line indicates where the head/neck ends and the body begins; the dashed black line indicates where the posterior of the animal begins; and the area of the body between the 2 lines is the trunk. The black arrow indicates the partial circle created by attaching a wire to 2 of the overhead video cameras, used to determine whether animal was within a body-length of the brush. with proximity as recorded by the RFID system. For this purpose, recordings of each heifer being detected as having (1) or not having (0) brush contact (video) were compared with being (1) or not being (0) in brush proximity (post-processed RFID data) for every second. Three measures were used to assess the relationship between the RFID and video-generated data: the coefficient of determination $\left(\mathrm{R}^{2}\right)$, whether if the slope was equal to 1 , and whether the intercept was equal to 0 (PROC REG in SAS software version 9.4; SAS Institute Inc.).

The sensitivity and specificity of the RFID system were calculated as follows for each heifer:

$$
\begin{gathered}
\text { sensitivity }=\mathrm{TP} / \mathrm{P}, \\
\text { and specificity }=\mathrm{TN} / \mathrm{N},
\end{gathered}
$$

where TP $=$ number of true positives (number of seconds where the heifer had brush contact according to the video with the RFID indicating brush proximity during the corresponding second); $\mathrm{P}=$ number of positives (number of seconds where the heifer had brush contact according to the video); $\mathrm{TN}=$ number of true negatives (number of seconds where the heifer did not have brush contact according to the video, with the RFID having a negative reading during the corresponding second); and $\mathrm{N}=$ number of negatives (number of seconds where the heifer did not have brush contact according to the video).

Video observations indicated that all heifers made at least some physical contact with the brush (Table 2). Across heifers, the majority of this contact involved the head/neck, in agreement with earlier studies (Georg and Totschek, 2001; DeVries et al., 2007). For this reason, we expected that tags placed on the ears would result in a high level of grooming detection, because 
Table 2. Total duration of time each heifer spent in brush contact, lying nearby, and standing nearby, along with total time observed (out of a maximum of $360 \mathrm{~min})^{1}$

\begin{tabular}{lcccc}
\hline Heifer & $\begin{array}{c}\text { Contact } \\
\text { time, min }\end{array}$ & $\begin{array}{c}\text { Lying nearby } \\
\text { time, min }\end{array}$ & $\begin{array}{c}\text { Standing nearby } \\
\text { time, min }\end{array}$ & $\begin{array}{c}\text { Total time, } \\
\text { min }\end{array}$ \\
\hline 316 & 11.4 & 49 & 98.4 & 334 \\
323 & 25.4 & 148 & 181.7 & 360 \\
330 & 16.9 & 0 & 39.0 & 368 \\
332 & 33.6 & 50 & 123.3 & 331 \\
333 & 37.5 & 63 & 131.3 & 334 \\
335 & 15.8 & 16 & 59.2 & 361 \\
336 & 18.2 & 81 & 121.8 & 334 \\
337 & 18.5 & 77 & 127.3 & 334 \\
350 & 19.8 & 0 & 55.0 & 360 \\
354 & 8.0 & 0 & 57.2 & 360 \\
358 & 13.1 & 0 & 36.2 & 334 \\
359 & 18.1 & 0 & 46.3 & 334 \\
368 & 3.7 & 0 & 20.3 & 334 \\
371 & 2.4 & 0 & 20.5 & 360 \\
373 & 6.1 & 0 & 19.0 & \\
374 & 20.3 & 0 & 38.7 & \\
\hline
\end{tabular}

${ }^{1}$ Data were recorded for 16 heifers, and based on video observations.

when contacting the brush, they would be within read range of the antennas. However, heifers varied in the ways that they used the brush, with trunk and posterior contact comprising the majority of contact on a proportional basis for certain individuals (Figure 3). Indeed, the ability of the RFID system to accurately detect brush contact, approximated by recording brush proximity, varied widely across individual heifers, as indicated by the sensitivity and specificity values (Table 3). Similarly, individual pigs that ate while lying down instead of while standing (as expected) had a higher level of false-negative readings, because while recumbent, the distance between tags and RFID antennas was increased relative to that of upright pigs (Maselyne et al., 2014). When designing these systems, it seems logical to plan for the most common anticipated behavioral patterns in choosing where to position tags and antennas but, as illustrated here, it is also important to consider that individual animals vary in the way that they interact with objects in their environment, and this can significantly alter the likelihood that they will be successfully detected by RFID.

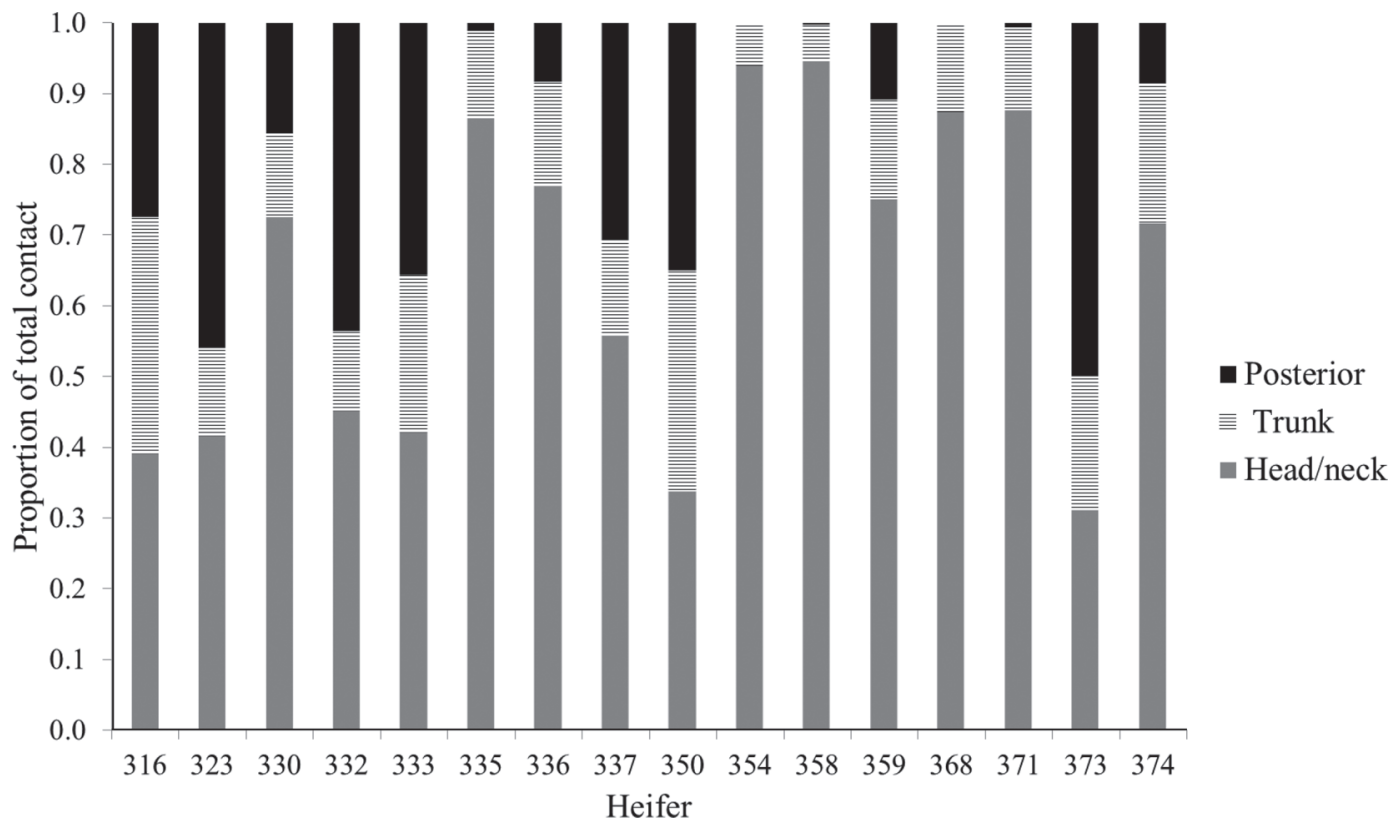

Figure 3. Proportion of total contact with brush on an individual-heifer basis involving head/neck (gray), trunk (striped), and posterior (black). 
We anticipated that heifers would primarily spend time near the brush while physically touching it. However, the majority of time that heifers were within a body length of the brush did not involve physical contact, but rather standing or lying nearby (Figure 4), sometimes for extended periods (Table 2), and therefore resulting in false-positive RFID readings (and hence low specificity; Table 3). It follows that the RFID system overestimated values relative to actual time spent in brush contact (Figure 5), and this relationship is supported by the relatively low $\mathrm{R}^{2}$ values along with a slope that is different from $1(P<0.01)$ and a non-zero intercept $(P<0.01)$. Similarly, evaluation of the linear relationship between brush contact and all 3 behaviors representing when the heifer was within 1 body length of the brush, including contact, standing nearby, and lying nearby (Figure 6) indicated that heifers spent more time near the brush relative to the amount of time actually grooming on it, with a moderate $\mathrm{R}^{2}$, slope $>1(P<0.01)$, and intercept $>0$ $(P=0.01)$. These relationships likely explain why the RFID system would overestimate grooming, because it detects animals within read range, regardless of whether they are actually using the device. Certain pigs monitored with RFID for feeding behavior were also noted to linger around the feed trough while not eating, which contributed to a high false-positive rate (Maselyne et al., 2014). Similar issues were found when comparing results from an RFID system that automatically recorded when mice touched or sniffed an object within close range versus these behaviors as observed on video (Catarinucci et al., 2014). Here too, researchers concluded that the RFID is unable to distinguish between an animal being near to an object and actually touching or sniffing it, and therefore overestimates the duration of these behaviors.

In conclusion, we expected that RFID recordings of brush proximity would provide a reasonable representation of grooming behavior. However, certain heifers spent a relatively large proportion of time near the grooming device even when not in contact (resulting in false-positive readings) and varied in how they used the brush, with some preferring to groom primarily the trunk and posterior parts of their bodies (resulting in false-negative readings). The implication is that RFID-based recording of brush proximity, although a potentially useful endeavor, does not provide an overall accurate approximation of grooming behavior and is not a reliable substitute for video recordings, as currently configured. It is possible that restricting the way in which cattle use the brush; for example, by constructing a radiofrequency-proof barrier around it such that only animals that are actually in contact with the device would be detected, could improve specificity. Similarly, sensitivity might be improved if cattle could access the brush only with their head and neck (e.g., through a head-gate), because ear-based tags would be more likely to be read during grooming. However, each of these barriers might reduce overall brush use if they required animals to exert extra effort to access

Table 3. Records of heifer being detected as having or not having brush contact (video) and being or not being in brush proximity (postprocessed RFID ${ }^{1}$ data) for every second of up to a 6 - $\mathrm{h}$ period $(0600-1200 \mathrm{~h})^{2}$

\begin{tabular}{|c|c|c|c|c|c|c|}
\hline Heifer & True positive ${ }^{3}$ & False positive ${ }^{4}$ & False negative $^{5}$ & True negative $^{6}$ & Sensitivity & Specificity \\
\hline 323 & 1,413 & 8,303 & 111 & 11,773 & 0.93 & 0.59 \\
\hline 330 & 875 & 337 & 138 & 20,730 & 0.86 & 0.98 \\
\hline 333 & 1,715 & 1,079 & 532 & 16,694 & 0.76 & 0.94 \\
\hline 335 & 941 & 774 & 9 & 19,936 & 0.99 & 0.96 \\
\hline 336 & 1,016 & 3,600 & 73 & 16,911 & 0.93 & 0.82 \\
\hline 354 & 477 & 1,645 & 0 & 19,478 & 1.00 & 0.92 \\
\hline 358 & 718 & 641 & 65 & 20,177 & 0.92 & 0.97 \\
\hline 359 & 815 & 688 & 269 & 18,269 & 0.75 & 0.96 \\
\hline 368 & 223 & 321 & 0 & 21,056 & 1.00 & 0.98 \\
\hline 371 & 146 & 1,909 & 0 & 17,966 & 1.00 & 0.90 \\
\hline 373 & 197 & 338 & 167 & 19,309 & 0.54 & 0.98 \\
\hline 374 & 1,106 & 1,054 & 110 & 19,330 & 0.91 & 0.95 \\
\hline
\end{tabular}

${ }^{1}$ Radiofrequency identification.

${ }^{2}$ For each heifer, the sensitivity and specificity of the post-processed RFID data (video = gold standard) were calculated.

${ }^{3}$ Number of seconds whereby both RFID and video detected animal at brush.

${ }^{4}$ Number of seconds whereby only RFID detected animal at brush.

${ }^{5}$ Number of seconds whereby only video detected animal at brush.

${ }^{6}$ Number of seconds whereby neither RFID nor video detected animal at brush. 


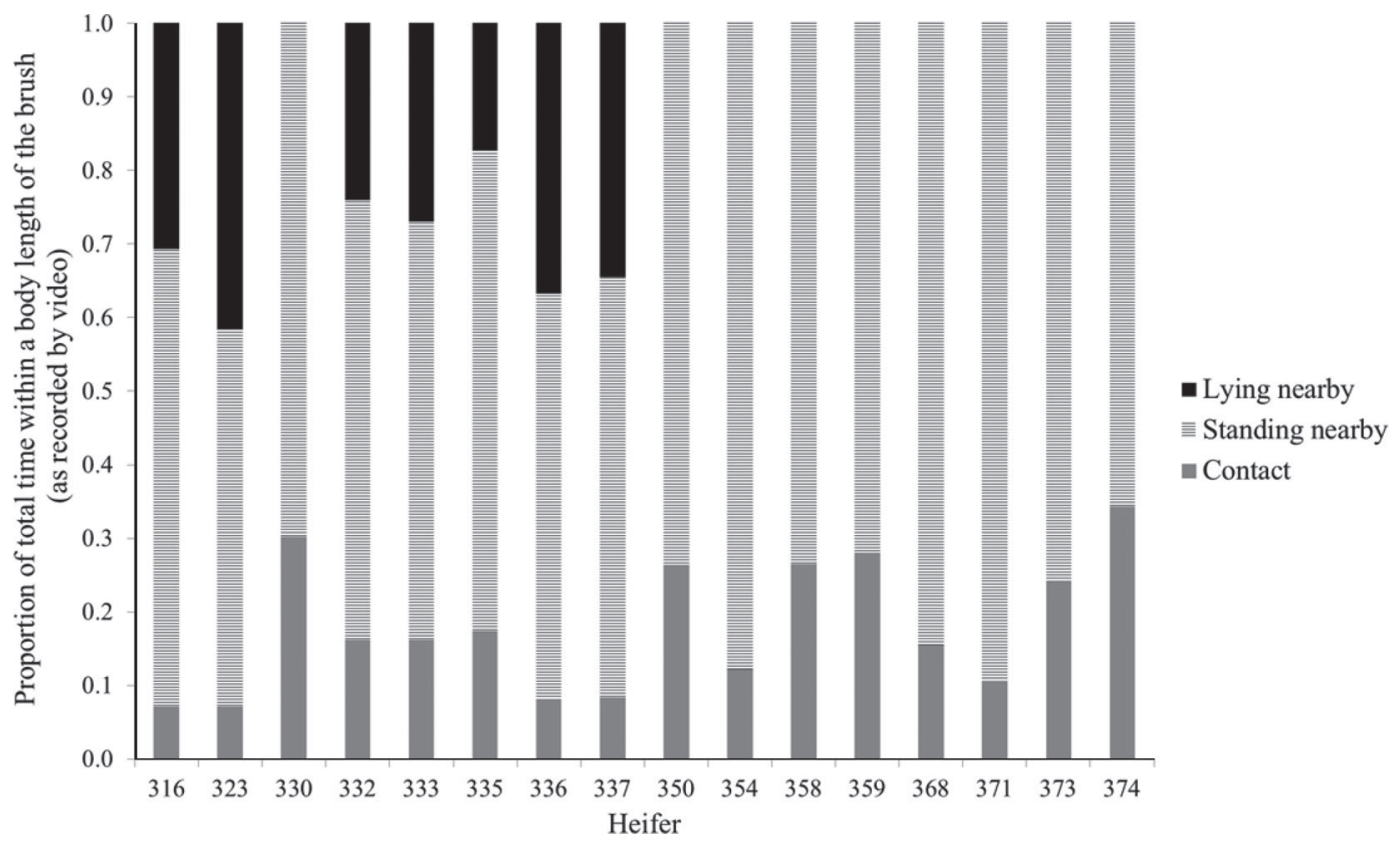

Figure 4. Proportion of total time spent in contact with (gray) or not in contact but still within a body length of the brush, either standing nearby (striped) or lying nearby (black), as recorded by video on an individual-heifer basis.

this resource or reduce motivation to use the device if unable to scratch their hind end or other hard-toreach locations. To this end, future work should con- sider alternate technologies or other RFID and brush configurations in an attempt to determine how to best automate measurement of grooming behavior.

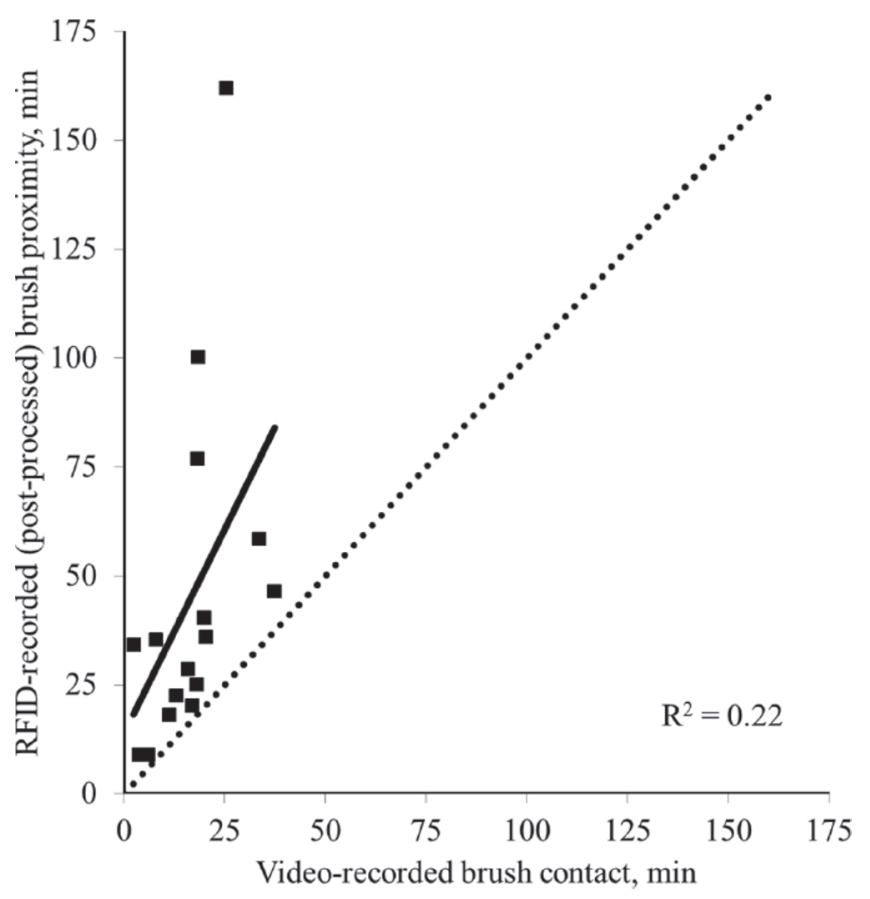

Figure 5. Representation of linear relationship between brush contact, as recorded by video (x-axis) and brush proximity, as indicated by radiofrequency identification (RFID) post-processed data (y-axis). A perfect 1:1 relationship is demonstrated by the dotted line.

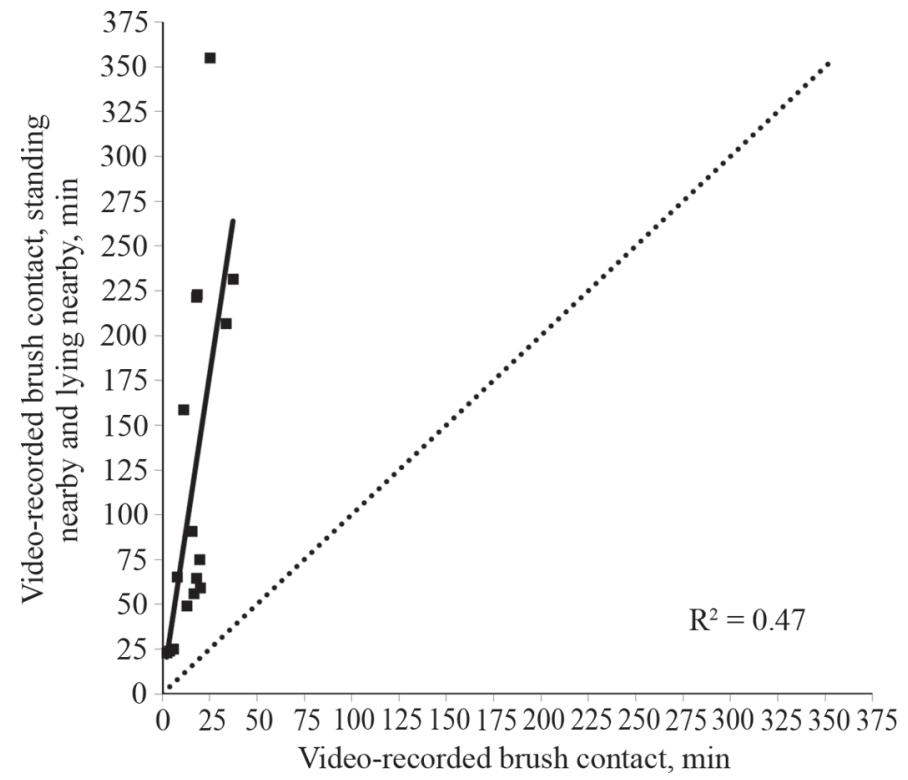

Figure 6. Representation of linear relationship between brush contact (x-axis) and all 3 behaviors representing when the heifer was within a body length of the brush, including contact, standing nearby, and lying nearby (y-axis), as recorded by video. A perfect 1:1 relationship is demonstrated by the dotted line. 


\section{ACKNOWLEDGMENTS}

This project was sponsored by National Research Initiative competitive grant no. 2011-68004-30367 from the USDA National Institute of Food and Agriculture (Washington, DC), and a research fellowship from the American Association of Bovine Practitioners (Auburn, $\mathrm{AL}$ ). The brushes were donated by DeLaval USA (Kansas City, MO). We gratefully acknowledge the infrastructure and support of the Department of Animal Science, College of Agricultural and Environmental Sciences and the California Agricultural Experiment Station of the University of California-Davis. Thank you to farm crew and feedlot personnel for assistance with animal and facility-related needs, Curt Finley for technical support, and the many undergraduate students who assisted with this work.

\section{REFERENCES}

Ambriz-Vilchis, V., N. S. Jessop, R. H. Fawcett, D. J. Shaw, and A. I. Macrae. 2015. Comparison of rumination activity measured using rumination collars against direct visual observations and analysis of video recordings of dairy cows in commercial farm environments. J. Dairy Sci. 98:1750-1758.

Borderas, T. F., A. M. de Passillé, and J. Rushen. 2008. Behavior of dairy calves after a low dose of bacterial endotoxin. J. Anim. Sci. 86:2920-2927.

Büchel, S., and A. Sundrum. 2014. Technical note: Evaluation of a new system for measuring feeding behavior of dairy cows. Comput. Electron. Agric. 108:12-16.

Catarinucci, L., R. Colella, L. Mainetti, L. Patrono, S. Pieretti, A. Secco, and I. Sergi. 2014. An animal tracking system for behavior analysis using radio frequency identification. Lab Anim. (NY) 43:321-327.

Chapinal, N., D. M. Veira, D. M. Weary, and M. A. G. von Keyserlingk. 2007. Technical Note: Validation of a system for monitoring individual feeding and drinking behavior and intake in grouphoused cattle. J. Dairy Sci. 90:5732-5736.
DeVries, T. J., M. Vankova, D. M. Veira, and M. A. G. von Keyserlingk. 2007. Short communication: Usage of mechanical brushes by lactating dairy cows. J. Dairy Sci. 90:2241-2245.

Georg, H., and K. Totschek. 2001. Investigation of an automatic brush for dairy cows. Landtechnik 56:260-261.

Hart, B. L. 1988. Biological basis of the behavior of sick animals. Neurosci. Biobehav. Rev. 12:123-137.

Mandel, R., H. R. Whay, E. Klement, and C. J. Nicol. 2016. Invited review: Environmental enrichment of dairy cows and calves in indoor housing. J. Dairy Sci. 99:1695-1715.

Mandel, R., H. R. Whay, C. J. Nicol, and E. Klement. 2013. The effect of food location, heat load, and intrusive medical procedures on brushing activity in dairy cows. J. Dairy Sci. 96:6506-6513.

Maselyne, J., W. Saeys, B. De Ketelaere, K. Mertens, J. Vangeyte, E. F. Hessel, S. Millet, and A. Van Nuffel. 2014. Validation of a high frequency radio frequency identification (HF RFID) system for registering feeding patterns of growing-finishing pigs. Comput. Electron. Agric. 102:10-18.

Matthews, S. G., A. L. Miller, J. Clapp, T. Plötz, and I. Kyriazakis. 2016. Early detection of health and welfare compromises through automated detection of behavioural changes in pigs. Vet. J. 217:43-51.

Mendes, E. D. M., G. E. Carstens, L. O. Tedeschi, W. E. Pinchak, and T. H. Friend. 2011. Validation of a system for monitoring feeding behavior in beef cattle. J. Anim. Sci. 89:2904-2910.

Newby, N. C., T. F. Duffield, D. L. Pearl, K. E. Leslie, S. J. LeBlanc, and M. A. G. von Keyserlingk. 2013. Short communication: Use of a mechanical brush by Holstein dairy cattle around parturition. J. Dairy Sci. 96:2339-2344.

Ott, S., C. P. H. Moons, M. A. Kashiha, C. Bahr, F. A. M. Tuyttens, D. Berckmans, and T. A. Niewold. 2014. Automated video analysis of pig activity at pen level highly correlates to human observations of behavioural activities. Livest. Sci. 160:132-137.

Toaff-Rosenstein, R., L. J. Gershwin, A. J. Zanella, and C. B. Tucker. 2016. The sickness response in steers with induced bovine respiratory disease before and after treatment with a non-steroidal antiinflammatory drug. Appl. Anim. Behav. Sci. 181:49-62.

Weary, D. M., J. M. Huzzey, and M. A. G. von Keyserlingk. 2009. Board-invited review: Using behavior to predict and identify ill health in animals. J. Anim. Sci. 87:770-777.

Wilson, S. C., F. M. Mitlöhner, J. Morrow-Tesch, J. W. Dailey, and J. J. McGlone. 2002. An assessment of several potential enrichment devices for feedlot cattle. Appl. Anim. Behav. Sci. 76:259-265. 